according to Australian guidelines, neither GAD7 nor HIV anxiety was independently associated with PrEP use. Among 406 PrEP-eligible men (26.2\%), PrEP users scored lower on GAD7 than did non-users $(\mathrm{OR}=0.95$; 95\%CI: 0.92-0.99) and PrEP use was independently associated with lower HIV anxiety $(\mathrm{aOR}=0.91 ; 95 \% \mathrm{CI}: 0.85-0.97)$.

Conclusion Among men who were eligible for PrEP, its use was independently associated with lower levels of anxiety in general, and of HIV anxiety specifically. PrEP use may help reduce anxiety among men who are at risk of HIV and may therefore offer perceived benefits in addition to avoiding HIV infection. This perceived benefit may be an important consideration in recommendations for PrEP use.

Disclosure No significant relationships.

\section{P446 USE OF ANTIBIOTIC PROPHYLAXIS FOR SEXUALLY TRANSMITTED INFECTIONS AMONG GAY AND BISEXUAL MEN IN AUSTRALIA}

${ }^{1}$ Vincent Cornelisse, ${ }^{2}$ Denton Callander, ${ }^{3}$ Christopher Fairley, ${ }^{4}$ Darren Russell. ${ }^{1}$ Melbourne Sexual Health Centre, Carlton, Australia; ${ }^{2}$ New York University, School of Medicine, New York, USA; ${ }^{3}$ Melbourne Sexual Health Centre, Melbourne, Australia; ${ }^{4}$ Queensland Health, Cairns Sexual Health Service, Cairns, Australia

\subsection{6/sextrans-2019-sti.530}

Background Antibiotic prophylaxis can reduce the risk of sexually transmitted infections (STIs), but concerns remain about its safety and feasibility of its implementation. We conducted an online survey to quantify current use of and interest in antibiotic prophylaxis among Australian gay and bisexual men. Methods From June to December 2018 our survey was promoted through gay community organisations and Melbourne Sexual Health Centre. The survey asked about demographics, sexual history, drug use, use of HIV pre-exposure prophylaxis (PrEP), history of STIs, use of antibiotic prophylaxis and attitudes towards antibiotic prophylaxis. We used logistic regression analyses to compare responses from respondents who had used antibiotic prophylaxis, respondents who had not used but were interested, and respondents who were not interested. Results A total of 517 survey responses were complete: 68 respondents $(13 \%)$ had previously used antibiotics to prevent STIs and $323(63 \%)$ expressed interest in using antibiotic prophylaxis. In univariate analyses, compared to respondents with no previous use and no interest, users of antibiotic prophylaxis were older $(M d n=43$ years vs $M d n=34$ years, $\mathrm{p}=0.018)$, had higher numbers of sexual partners $(M=14$ vs $M=5$, $\mathrm{p}=0.002)$, were more likely to report recent group sex $(64.7 \%$ vs $42.1 \%, \mathrm{p}=0.003)$, to be using HIV PrEP $(66.2 \%$ vs $35.7 \%, \mathrm{p}<0.001)$, and report a recent bacterial STI diagnosis $(57.4 \%$ vs $31.0 \%, \mathrm{p}<0.001)$. However, in multivariate analysis, users of antibiotic prophylaxis were not more likely to report a recent bacterial STI diagnosis $(\mathrm{aOR}=1.65,95 \% \mathrm{CI}$ 0.8-3.5), after adjusting for age, sexual partner numbers, drug use, and PrEP use.

Conclusion A majority of respondents expressed interest in antibiotic prophylaxis and $13 \%$ had used antibiotic prophylaxis. Users of antibiotic prophylaxis reported more STI risk factors and had more bacterial STIs than non-users. However, the use of antibiotic prophylaxis was not independently associated with a higher risk of STI diagnosis.

Disclosure No significant relationships.

\section{P447 AWARENESS AND ACCEPTABILITY OF PRE-EXPOSURE PROPHYLAXIS AMONG MSM: RESULTS FROM SCOTLAND'S GAY BAR SURVEY}

${ }^{1}$ Jamie Frankis*, ${ }^{2}$ Lisa Mcdaid, ${ }^{3}$ Lesley Wallace, ${ }^{4}$ David Goldberg, ${ }^{2}$ Paul Flowers. ${ }^{1}$ Glasgow Caledonian University, Glasgow, UK; ${ }^{2}$ University of Glasgow, MRC/CSO Social and Public Health Sciences Unit, Glasgow, UK; ${ }^{3}$ Health Protection Scotland, QU, UK; ${ }^{4}$ Health Protection Scotland, QE, UK

\subsection{6/sextrans-2019-sti.531}

Background Scotland was the first country to introduce free criterion-based PrEP for all citizens. So far, uptake has been almost exclusively among MSM. Herein, we examine PrEP awareness and acceptability among MSM in Scotland from a national level behavioural surveillance project.

Methods Time and location sampling was used to survey $\mathrm{n}=972$ MSM across the commercial gay scenes of Scotland's two largest cities.

Results $5.4 \%$ of participants were HIV+. 94.6\% were HIV-/ untested, of whom, $4.5 \%$ were on PrEP. Most of the remaining men had heard of PrEP (81.7\%). Multivariate logistic regression suggested that gay men $(\mathrm{OR}=2.76)$, men aged 26$35(\mathrm{OR}=2.38)$ and $36-45(\mathrm{OR}=2.31)$, men who used the gay scene $\geq=$ twice a month $(\mathrm{OR}=2.19)$, reported an HIV test in the last year $(\mathrm{OR}=1.96)$ or an STI other than HIV diagnosed in the last year $(\mathrm{OR}=3.47)$ were significantly $(p<0.05)$ more likely to have heard of PrEP. Around one third $(31.1 \%)$ of HIV-/untested men said they were likely to use PrEP now it is available. Multivariate logistic regression suggested that gay men $(\mathrm{OR}=2.23)$, younger men $(18-25$, $\mathrm{OR}=2.73 ; 26-35, \mathrm{OR}=2.48 ; 36-45, \mathrm{OR}=2.00)$, single men $(\mathrm{OR}=2.08)$, men who use the gay scene $\geq=$ twice a month $(\mathrm{OR}=1.51)$, men who report high risk condomless anal intercourse $(\mathrm{OR}=1.61)$, an $\mathrm{HIV}$ test in the last year $(\mathrm{OR}=1.95)$ or an STI other than HIV diagnosed in the last year $(\mathrm{OR}=1.61)$ were significantly more likely to consider using PrEP in the future.

Conclusion PrEP awareness among MSM in Scotland is high, but disparities in awareness remain along traditional indicators of inequality. Findings suggest substantial interest in PrEP, particularly among those most likely to benefit although other issues may be more indicative of use than pure behavioural risk. As the biobehavioural HIV risk management strategies now available to MSM require high levels of health literacy, health promotion strategies must focus on enabling men to negotiate these complexities.

Disclosure No significant relationships. 